ISSN : 0825-8643

\title{
Updating Claims in Bankruptcy Problems
}

M. Josune ALBIZURI

Justin LEROUX

José Manuel ZARZUELO

\section{Cahier de recherche $n^{\circ}$ IEA-08-08}

August 2008

Copyright (C) 2008 HEC Montréal.

Tous droits réservés pour tous pays. Toute traduction ou toute reproduction sous quelque forme que ce soit est interdite. Les textes publiés dans la série des Cahiers de recherche HEC n'engagent que la responsabilité de leurs auteurs.

La publication de ce Cahier de recherche a été rendue possible grâce à des subventions d'aide à la publication et à la diffusion de la recherche provenant des fonds de l'École des HEC.

Direction de la recherche, HEC Montréal, 3000, chemin de la Côte-Sainte-Catherine, Montréal (Québec) Canada H3T 2 A7. 
Institut d'économie appliquée

HEC Montréal

3000 chemin de la Côte-Sainte-Catherine

Montréal (Québec) H3T 2A7

Canada

http://www.hec.ca/iea

iea.info@hec.ca

Copyright (c) 2008 HEC Montréal.

Tous droits réservés pour tous pays. Toute traduction ou toute reproduction sous quelque forme que ce soit est interdite sans l'autorisation expresse de HEC Montréal.

Les textes publiés dans la série des Cahiers de recherche HEC Montréal n'engagent que la responsabilité de leurs auteurs.

ISSN : 0825-8643 


\title{
UPDATING CLAIMS IN BANKRUPTCY PROBLEMS
}

\author{
M. J. ALBIZURI, J. LEROUX AND J. M. ZARZUELO
}

\begin{abstract}
We reexamine the consistency axiom in bankruptcy problems and propose arguments in favor of an alternative definition of a reduced problem. The classical definition updates the size of the estate while keeping agents' claims unaffected. Instead, we suggest updating agents' claims along with the estate. The resulting consistency axiom characterizes the well-known Random Arrival rule as the unique bilaterally consistent extension of the Contested Garment rule to many agents. We also establish that our definition of a reduced bankruptcy problem corresponds to the definition of a reduced TU game proposed in Hart \& Mas-Colell (Econometrica, 1989). JEL classification: C7.
\end{abstract}

\section{INTRODUCTION}

A bankruptcy problem refers to a situation in which a group of individuals have rights over an estate, but the estate is not large enough to cover their joint claims. Such problems appear for instance when a firm declares bankruptcy and creditor claims cannot be satisfied. From a normative standpoint, the main issue is to find rules which specify a division of the estate according to the claims in a fair way via the formulation of desirable properties, or axioms, which transpose philosophical principles to the problem at hand. Early work by O'Neill (1982) lays ground to the formalism we use here to study bankruptcy problems. For an extensive review on this topic the reader may also consult Thomson (2003).

A principle which has played a significant role in many economic allocation problems is that of consistency. According to this principle, if a

Date: April 3, 2008.

Key words and phrases. Bankruptcy Problems, Consistency, Cooperative Games.

M. J. Albizuri: elpalirm@bs.ehu.es; J. M. Zarzuelo: elpzazaj@bs.ehu.es, Fac. of Economics and Business Administration. Av. Lehendakari Aguirre, 83; 48015 Bilbao, Spain J. Leroux: justin.leroux@hec.ca, HEC Montréal and CIRPÉE, 3000 chemin de la Côte Sainte-Catherine, Montréal, QC H3T 2A7, Canada.

We wish to thank Jérémy Laurent-Lucchetti for his comments to highlight the motivations of the paper. This research has been partially supported by the Basque Government (project GICO07/155-IT-293-07), DGES Ministerio de Educación y Ciencia (project SEJ 2006-05455), and the Fonds Québécois de la Recherche sur la Société et la Culture. 
subgroup of agents decides to leave the others, the rewards the remaining agents obtain by (re)applying the same sharing rule among themselves should be unchanged. In bankruptcy problems the consistency axiom, or its weaker version of bilateral consistency, has been used in the characterization of several rules rules; see for instance Aumann and Maschler (1985), or Young (1987) among others. Customarily, in the reduced problem the residual estate is defined as the original estate minus the sum of the rewards paid to the departing agents, while all claims remain the same. So in the reduced situation the estate is updated, while claims are not.

One can argue that this definition only partially handles the problem reduction by omitting to update agents' claims. Indeed, one can argue that when agents face their reduced problem, they have already conceded part of their claims to the departing agents, and vice versa. If so, their claims in the reduced problem should be updated, and may differ from their original ones. For instance, when agent $i$ departs with her share, one may argue that she forgoes a portion of her claim on the remainder of the estate she is not receiving. To be consistent with this argument, one must further consider that the remaining agents also give up a portion of their own claim on the share of the estate agent $i$ has secured. Following this intuition, we propose an alternative definition of the reduced problem in which the estate and the claims are simultaneously updated.

To calculate the share of the claim a departing agent $i$ concedes to a remaining agent $k$ we proceed as follows. First, partition the estate into two parts: agent $i$ 's claim and the rest of the estate unclaimed by agent $i$, if any. The other agents can negotiate this remainder between them in a subproblem by applying the sharing rule in effect. The share of agent $k$ in this subproblem is not conceded as part of agent $i$ 's claim. Therefore, the part of his claim agent $i$ concedes to agent $k$ is the difference between agent $k$ 's share in the original problem and his agent $k$ 's share in this subproblem. Hence, such updated claims are typically less than the original ones. This definition of updated claims leads to a definition of a reduced bankruptcy problem different from the traditional one.

A key result in the literature on bankruptcy problems is that the Talmudic solution is the unique bilaterally consistent - in the usual sense - of the Contested Garment rule (Aumann \& Maschler, 1985). By contrast, using our definition of a reduced problem, we characterize another important sharing method, the Random Arrival rule (O'Neill, 1982), as the unique bilaterally consistent extension of the Contested Garment rule to many agents (Theorem 2). 
Finally, in Section 4 we examine the relationship between cooperative TU games and bankruptcy problems. TU games are particularly suitable to examining bankruptcy problems, and there exists a natural way to associate every bankruptcy problem with a TU game. The consistency property has also received considerable attention in the context of TU games, and several definitions of reduced games exist in this literature as well. We shall focus on the definitions proposed by Davis \& Maschler (1965), and Hart \& Mas-Colell (1989) and highlight the relationship between these definitions of a reduced TU game and the definitions of reduced bankruptcy problem. Aumann \& Maschler (1985) establishes that the traditional way of reducing a bankruptcy problem corresponds to the Davis \& Maschler reduced game. In turn, we find (Theorem 3) that the definition of a reduced bankruptcy problem we introduced here corresponds to the Hart \& Mas-Colell reduced game for bilateral reduced problems. However, in general the Hart \& MasColell reduced game of a bankruptcy problem is not a game associated to any bankruptcy game for any symmetric rule.

In other words, our results suggest that the dichotomy of the consistency axiom which is found in cooperative games, but also in cost-sharing problems ${ }^{1}$, arises in bankruptcy problems as well.

\section{Preliminaries}

Let $U$ denote a set of potential agents. Given a non-empty finite subset $N$ of $U$, by $\mathbf{R}^{N}$ denote the $|N|$-dimensional Euclidean space with axes labeled by the members of $N$, and $\mathbf{R}_{+}^{N}=\left\{x \in \mathbf{R}^{N}: x_{i} \geq 0\right\}$. If $S \subseteq N, S \neq \emptyset$, and $x=\left(x_{i}\right)_{i \in N} \in \mathbf{R}^{N}$, then $x_{S}$ denotes the projection of $x$ onto $\mathbf{R}^{S}$, i. e., $x_{S}=\left(x_{i}\right)_{i \in S} \in \mathbf{R}^{S}$, and $x(S)=\sum_{i \in N} x_{i}$. Finally, if $x \in \mathbf{R}$ we denote $x_{+}=\max \{x, 0\}$.

A triple $(N, E, c)$ is called a bankruptcy problem, if $N$ is a non-empty finite subset of $U$ (the set of agents involved in the problem), $E \in \mathbf{R}_{+}$(the estate), and $c \in \mathbf{R}_{+}^{N}$ (the vector of claims) is such that $\sum_{i \in N} c_{i} \geq E$. We will denote $\bar{c}_{i}=\min \left\{E, c_{i}\right\}$.

Let $\mathcal{B}^{U}$ denote the set of all bankruptcy problems with the foregoing properties.

\footnotetext{
${ }^{1}$ See, e.g., Sudhölter (1998) for a comparison and Leroux (2007) for a discussion regarding the Davis \& Maschler and the Hart \& Mas-Colell interpretations of the consistency axiom in the cost-sharing literature and their respective characterizations of the nucleolus and the Shapley rule.
} 
A bankruptcy rule $\sigma$ associates with each $(N, E, c) \in \mathcal{B}^{U}$ a vector $\sigma(N, E, c) \in$ $\mathbf{R}_{+}^{N}$ satisfying:

(a) $\sum_{i \in N} \sigma_{i}(N, E, c)=E$,

(b) $\sigma_{i}(N, E, c) \leq c_{i}$,

(c) $\sigma(N, E, c)=\sigma(N, E, \bar{c})$.

The first condition is an efficiency property stating that a cost sharing rule must exactly allocate the total value of the estate. The second property requires that no agent be awarded more than her claim. Finally, the third property is one of independence of claims higher than the estate according to which agents' claims are only valid to the extent that they do not exceed the total value of the estate.

In the most simple case where only 2 agents are involved, let us consider the following principle that can be traced back to the Babylonian Talmud: "Each claimant $i$ concedes $\left(E-\bar{c}_{i}\right)_{+}$to the other, and the remaining is equally divided". This is known as the Contested Garment principle (Aumann \& Maschler, 1985). Accordingly define for each $(N, E, c) \in \mathcal{B}^{U}$ such that $|N|=2$, the $C G$ rule, for each $i \in N$ by

$$
C G_{i}(N, E, c)=\left(E-c_{j}\right)_{+}+\frac{E-\left(E-c_{i}\right)_{+}-\left(E-c_{j}\right)_{+}}{2}=\frac{E+\bar{c}_{i}-\bar{c}_{j}}{2} .
$$

Several rules have been suggested for more general problems extending the CG rule to many agents (for a survey consult Thomson, 2003). For instance the Talmud rule, T, introduced in Aumann \& Maschler (1985), is defined as follows:

For each $(N, E, c) \in \mathcal{B}$ and each $i \in N$,

1. If $\sum_{k \in N}\left(c_{k} / 2\right) \geq E$, then $T_{i}(N, E, c) \equiv \min \left\{c_{i} / 2, \lambda\right\}$, where $\lambda$ is chosen so that $\sum_{k \in N} \min \left\{c_{k} / 2, \lambda\right\}=E$,

2. If $\sum_{k \in N}\left(c_{k} / 2\right) \leq E$, then $T_{i}(N, E, c) \equiv c_{i}-\min \left\{c_{i} / 2, \lambda\right\}$, where $\lambda$ is chosen so that $\sum_{k \in N}\left(c_{k}-\min \left\{c_{i} / 2, \lambda\right\}\right)=E$.

We will mainly focus on a rule proposed by O'Neill (1982) commonly called the Random Arrival rule, which we denote by $R A$. To define this rule formally let $\Pi^{N}$ denote the class of permutations of $N$. For convenience, denote for each $(N, E, c) \in \mathcal{B}^{U}, i \in N$, and $\pi \in \Pi^{N}$ :

$$
M C_{i}((N, E, c), \pi):=M C_{i}(\pi)=\min \left\{\bar{c}_{i}, \max \left(E-\sum_{\substack{j \in N: \\ \pi(j)<\pi(i)}} \bar{c}_{j}\right)_{+}\right\} .
$$

The permutation $\pi$ represents an ordering on $N$. The real number $M C_{i}(\pi)$ has the following interpretation. The first individual in this ordering receives 
his claim, or the entire estate if his claim is greater. The next agent is given his claim, or the estate that is left if his claim is greater, and so on. Then $M C_{i}(\pi)$ is what agent $i$ would receive if the agents were paid by using this method. If we assume that all the orderings $\pi$ are equally likely, then the expected payoff of agent $i$ is precisely what the $R A$ rule prescribes. That is:

$$
R A_{i}(N, E, c):=\mathbf{E}\left(M C_{i}\right)=\frac{1}{n !} \sum_{\pi \in \Pi^{N}} M C_{i}(\pi) .
$$

\section{Consistency}

The consistency axiom has played a significant role in the axiomatization of bankruptcy rules (see Thomson, 2006, for a comprehensive survey). Consistency can be described as follows. Let $\sigma$ be a rule applied to a specific bankruptcy problem. This rule is said to be consistent if whenever a subgroup of agents are paid according to $\sigma$-thus leaving the other agents with a reduced problem - the payoffs of the remaining agents do not change after (re)applying $\sigma$ to the reduced problem. Several ways of defining a reduced problem exist, in turn giving rise to different interpretations of the consistency axiom.

Given a rule $\sigma,(N, E, c) \in \mathcal{B}^{U}$, and a coalition $S \subset N$, define

$$
E^{S, \sigma}=E-\sum_{k \in N \backslash S} \sigma_{k}(N, E, c) .
$$

That is, $E^{S, \sigma}$ denotes what is left to the members of $S$ after paying to the rest of the agents according to $\sigma$. The traditional definition of the reduced game of a coalition $S \subseteq N$ is written as $\left(N, E^{S, \sigma}, c_{S}\right)$. Note that the size of the estate is updated but claims are not.

Consequently, following Aumann \& Maschler (1985) a rule $\sigma$ is called $C G$-bilateral consistent if for any $(N, E, c) \in \mathcal{B}^{U}$, and every $S \subseteq N$ such that $|S|=2$, its restriction to $S$ coincides with the Contested Garment rule on the reduced problem of $S$ :

$$
\sigma_{S}(N, E, c)=C G\left(S, E^{S, \sigma}, c_{S}\right) .
$$

Theorem 1. (Aumann \& Maschler, 1985) The Talmud rule is the unique $C G$-bilateral consistent solution.

In the definition of CG-bilateral consistency presented above, it is assumed that agents keep their claims invariant in the reduced problem. However, one can argue that this definition only partially handles the problem reduction by omitting to update agents' claims. Indeed, one can argue that when agents face their reduced problem, they have already conceded part 
of their claims to the departing agents. If so, their claims in the reduced problem should be updated, and may differ from the original claims. Alternatively, when agent $i$ departs with her share, one may argue that she forgoes a portion of her claim on the remainder of the estate she is not receiving. In other words, her claim has been "truncated" to equal exactly her share on the residual problem where she is alone and her residual estate equals her allotted share. To be consistent with this argument, one must also consider that the remaining agents give up a portion of their own claim on the share of the estate agent $i$ has secured via $\sigma$.

Formally, we suggest the following way to update claims. To calculate the updated claims of agents $k \in N \backslash\{i\}$ after agent $i$ has left with her share we proceed as follows. First, partition the estate into two parts: $\bar{c}_{i}$, i.e. agent $i$ 's claim, and $E-\bar{c}_{i}$, which is the portion of the estate uncontested by agent $i$. On the latter portion of the estate, every agent $k \in N \backslash\{i\}$ should obtain $\sigma_{k}\left(N \backslash\{i\}, E-\bar{c}_{i}, \bar{c}_{N \backslash\{i\}}\right)$. The remainder of agent $k$ 's payoff, $\sigma_{k}(N, E, \bar{c})-$ $\sigma_{k}\left(N \backslash\{i\}, E-\bar{c}_{i}, \bar{c}_{N \backslash\{i\}}\right)$, is then obtained from $\bar{c}_{i}$. More generally, when considering the reduced problem of a coalition $S \subset N$, we define the updated claim of agent $i \in S$ as follows:

$$
c_{i}^{S, \sigma}=\bar{c}_{i}-\sum_{k \in N \backslash S}\left(\sigma_{k}(N, E, \bar{c})-\sigma_{k}\left(N \backslash\{i\}, E-\bar{c}_{i}, \bar{c}_{N \backslash\{i\}}\right)\right) .
$$

Remark 1. Notice that if $S=\{i, j\}$ then:

a) $c_{i}^{S, \sigma}+c_{j}^{S, \sigma} \geq E^{S, \sigma}$, so $\left(S, E^{S, \sigma}, c^{S, \sigma}\right)$ is a bankruptcy problem,

b) $0 \leq c_{i}^{S, \sigma} \leq E^{S, \sigma}$, hence $c_{i}^{S, \sigma}=\overline{c_{i}^{S, \sigma}}$, and

c) $c_{i}^{\{i\}, \sigma}=\sigma_{i}(N, E, \bar{c})$.

We say that a rule $\sigma$ is $C G$-bilateral consistent* if for every $(N, E, c) \in \mathcal{B}^{U}$ and every $S \subseteq N$ such that $|S|=2$ it holds

$$
\sigma_{S}(N, E, c)=C G\left(S, E^{S, \sigma}, c^{S, \sigma}\right) .
$$

As it turns out, this version of bilateral consistency where claims are updated in the subproblem characterizes the RA rule:

Theorem 2. The RA rule is the unique CG-bilateral consistent* solution.

This theorem is consequence of the two following propositions.

Proposition 1. There is at most one CG-bilateral consistent ${ }^{*}$ solution.

Proof. Let $\sigma$ and $\psi$ be two CG-bilateral consistent* rules. We check that both coincide on every bankruptcy problem $(N, E, c) \in \mathcal{B}^{U}$ by induction on $|N|$, with $|N|=2$ being the hypothesis. 
We assume by contradiction that $x=\sigma(N, E, c) \neq \psi(N, E, c)=y$ for some $(N, E, c) \in \mathcal{B}^{U}$ with $|N|>2$. By efficiency, there exist $S=\{i, j\} \subset N$ such that $x_{i}>y_{i}$ and $x_{j}<y_{j}$. By definition, for any $\ell \in S$ it holds:

$$
\begin{aligned}
c_{\ell}^{S, \sigma} & =\bar{c}_{\ell}-\sum_{k \in N \backslash S}\left(\sigma_{k}(N, E, \bar{c})-\sigma_{k}\left(N \backslash\{\ell\}, E-\bar{c}_{\ell}, \bar{c}_{N \backslash\{\ell\}}\right)\right) \\
& =\bar{c}_{\ell}-E+x_{i}+x_{j}+\sum_{k \in N \backslash S} \sigma_{k}\left(N \backslash\{\ell\}, E-\bar{c}_{\ell}, \bar{c}_{N \backslash\{\ell\}}\right) .
\end{aligned}
$$

Similarly,

$$
c_{\ell}^{S, \psi}=\bar{c}_{\ell}-E+y_{i}+y_{j}+\sum_{k \in N \backslash S} \psi_{k}\left(N \backslash\{\ell\}, E-\bar{c}_{\ell}, \bar{c}_{N \backslash\{\ell\}}\right) .
$$

By the induction hypothesis, we have:

$$
\sigma_{k}\left(N \backslash\{\ell\}, E-\bar{c}_{\ell}, \bar{c}_{N \backslash\{\ell\}}\right)=\psi_{k}\left(N \backslash\{\ell\}, E-\bar{c}_{\ell}, \bar{c}_{N \backslash\{\ell\}}\right) .
$$

Hence

$$
c_{i}^{S, \sigma}-c_{i}^{S, \psi}=\left(x_{i}+x_{j}\right)-\left(y_{i}+y_{j}\right)=c_{j}^{S, \sigma}-c_{j}^{S, \psi},
$$

and consequently

$$
c_{i}^{S, \sigma}-c_{j}^{S, \sigma}=c_{i}^{S, \psi}-c_{j}^{S, \psi}
$$

which by Remark 1 implies

$$
\overline{c_{i}^{S, \sigma}}-\overline{c_{j}^{S, \sigma}}=\overline{c_{i}^{S, \psi}}-\overline{c_{j}^{S, \psi}}
$$

But then by definition of the $C G$ rule, we have for each $\ell \in S$ :

$$
C G_{\ell}\left(S, E^{S, \sigma}, c^{S, \sigma}\right)=C G_{\ell}\left(S, E^{S, \psi}, c^{S, \psi}\right)+\frac{E^{S, \sigma}-E^{S, \psi}}{2},
$$

that is

$$
x_{\ell}=y_{\ell}+\frac{E^{S, \sigma}-E^{S, \psi}}{2},
$$

which is in contradiction with the fact that $x_{i}>y_{i}$ and $x_{j}<y_{j}$.

To prove the next proposition the following lemma will be useful. We introduce the following notation: Given a permutation $\pi$ of $N$, and $\ell \in N$, we will denote by $\pi^{\ell}$ the permutation such that:

i) $\pi(k)<\pi\left(k^{\prime}\right)$ implies $\pi^{\ell}(k)<\pi^{\ell}\left(k^{\prime}\right)$ for every $k, k^{\prime} \neq \ell$, and

ii) $\pi^{\ell}(k)>\pi^{\ell}(\ell)$ for every $k \neq \ell$.

(Thus, in the ordering $\pi^{\ell}$, agents others than $\ell$ keep their relative order invariant while $\ell$ becomes first). Also we will denote by $\Pi^{\ell}$ the set of permutations in which $\ell$ comes first; i.e. $\Pi^{\ell}=\left\{\pi \in \Pi^{N}: \pi(k)>\pi(\ell)\right.$ for every $k \neq$ $\ell\}$.

Moreover if $\pi \in \Pi^{N}$, and $i, j \in N$ we denote by $\pi_{i j}$ the permutation in $\Pi^{N}$ such that: 
i) $\pi_{i j}(i)=\pi(j)$ and $\pi_{i j}(j)=\pi(i)$, and

ii) $\pi_{i j}(k)=\pi(k)$ for every $k \neq i, j$.

(i.e., $\pi_{i j}$ interchanges the location of $i$ and $j$ in the ordering $\pi$ while leaving the rest of the ordering unaltered).

Finally we denote $\pi_{i j}^{i}=\left(\pi_{i j}\right)^{i}$.

Lemma 1. If $\pi \in \Pi^{N}$ and $i, j \in N$ are such that $\pi(i)<\pi(j)$, then

$$
M C_{i}(\pi)-M C_{i}\left(\pi^{j}\right)=M C_{j}\left(\pi_{i j}\right)-M C_{j}\left(\pi_{i j}^{i}\right)
$$

Proof. Notice that for every $\ell \in N$, and every $\pi \in \Pi^{N}$ it holds

$$
M C_{\ell}(\pi)=\min \left(E, \sum_{\pi(k) \leq \pi(\ell)} c_{k}\right)-\min \left(E, \sum_{\pi(k)<\pi(\ell)} c_{k}\right)
$$

Then we have

$$
\begin{aligned}
& M C_{i}(\pi)- M C_{i}\left(\pi^{j}\right)= \\
& {\left[\min \left(E, \sum_{\pi(k) \leq \pi(i)} c_{k}\right)-\min \left(E, \sum_{\pi(k)<\pi(i)} c_{k}\right)\right] } \\
&-\left[\min \left(E, \sum_{\pi^{j}(k) \leq \pi^{j}(i)} c_{k}\right)-\min \left(E, \sum_{\pi^{j}(k)<\pi^{j}(i)} c_{k}\right)\right] \\
&= {\left[\min \left(E, \sum_{\pi_{i j}^{i}(k)<\pi_{i j}^{i}(j)} c_{k}\right)-\min \left(E, \sum_{\pi_{i j}(k)<\pi_{i j}(j)} c_{k}\right)\right] } \\
&- {\left[\min \left(E, \sum_{\pi_{i j}^{i}(k) \leq \pi^{i}(j)} c_{k}\right)-\min \left(E, \sum_{\pi_{i j}(k) \leq \pi_{i j}(j)} c_{k}\right)\right] } \\
&=M C_{j}\left(\pi_{i j}\right)-M C_{j}\left(\pi_{i j}^{i}\right) .
\end{aligned}
$$

And the proof is complete.

Proposition 2. RA is CG-bilateral consistent*.

Proof. Let $S=\{i, j\} \subset N$, and $\pi$ be any permutation of $N$, then we have:

- If $\pi(i)>\pi(j)$ : then $M C_{i}(\pi)=M C_{i}\left(\pi^{j}\right)$.

- If $\pi(i)<\pi(j)$ : then $M C_{i}(\pi)=M C_{i}\left(\pi^{j}\right)+\left(M C_{i}(\pi)-M C_{i}\left(\pi^{j}\right)\right)$. 
Hence

$$
\begin{gathered}
R A_{i}(N, E, c)=\frac{1}{n !} \sum_{\substack{\pi \in \Pi: \\
\pi(i)>\pi(j)}} M C_{i}(\pi)+\frac{1}{n !} \sum_{\substack{\pi \in \Pi: \\
\pi(i)<\pi(j)}} M C_{i}(\pi) \\
=\frac{1}{n !} \sum_{\substack{\pi \in \Pi: \\
\pi(i)>\pi(j)}} M C_{i}\left(\pi^{j}\right)+\frac{1}{n !} \sum_{\substack{\pi \in \Pi: \\
\pi(i)<\pi(j)}}\left(M C_{i}\left(\pi^{j}\right)+\left(M C_{i}(\pi)-M C_{i}\left(\pi^{j}\right)\right)\right. \\
=\frac{1}{(n-1) !} \cdot \sum_{\pi \in \Pi^{j}} M C_{i}(\pi)+\frac{1}{n !} \sum_{\substack{\pi \in \Pi !: \\
\pi(i)<\pi(j)}}\left(M C_{i}(\pi)-M C_{i}\left(\pi^{j}\right)\right)
\end{gathered}
$$

Analogously

$$
R A_{j}(N, E, c)=\frac{1}{(n-1) !} \cdot \sum_{\pi \in \Pi^{i}} M C_{j}(\pi)+\frac{1}{n !} \sum_{\substack{\pi \in \Pi: \\ \pi(j)<\pi(i)}}\left(M C_{j}(\pi)-M C_{j}\left(\pi^{i}\right)\right) .
$$

By Lemma 1 and the definition of the $R A$ rule we have, and the definition of the $C G$ rule

$$
\begin{gathered}
R A_{i}(N, E, c)-R A_{j}(N, E, c)= \\
\frac{1}{(n-1) !} \cdot\left(\sum_{\pi \in \Pi^{j}} M C_{i}(\pi)-\sum_{\pi \in \Pi^{i}} M C_{j}(\pi)\right)= \\
R A_{i}\left(N \backslash\{j\}, E-\bar{c}_{j}, c_{N \backslash\{j\}}\right)-R A_{j}\left(N \backslash\{i\}, E-\bar{c}_{i}, c_{N \backslash\{i\}}\right)= \\
E-\bar{c}_{j}-\sum_{k \in N \backslash S} R A_{k}\left(N \backslash\{j\}, E-\bar{c}_{j}, \bar{c}_{N \backslash\{j\}}\right)-\left(E-\bar{c}_{i}\right)+\sum_{k \in N \backslash S} R A_{k}\left(N \backslash\{i\}, E-\bar{c}_{i}, \bar{c}_{N \backslash\{i\}}\right) \\
=c_{i}^{S, R A}-c_{j}^{S, R A}=C G_{i}\left(N, E^{S, R A}, c^{S, R A}\right)-C G_{j}\left(N, E^{S, R A}, c^{S, R A}\right) .
\end{gathered}
$$

Hence

$$
R A_{i}(N, E, c)-R A_{j}(N, E, c)=R A_{i}\left(N, E^{S, R A}, c^{S, \sigma}\right)-R A_{j}\left(N, E^{S, R A}, c^{S, \sigma}\right)
$$

On other hand by efficiency

$$
R A_{i}(N, E, c)+R A_{j}(N, E, c)=R A_{i}\left(N, E^{S, R A}, c^{S, \sigma}\right)+R A_{j}\left(N, E^{S, R A}, c^{S, \sigma}\right) .
$$

From the last two equalities

$R A_{i}(N, E, c)=R A_{i}\left(N, E^{S, R A}, c^{S, \sigma}\right)$ and $R A_{j}(N, E, c)=R A_{j}\left(N, E^{S, R A}, c^{S, \sigma}\right)$, and the proof is done. 


\section{Cooperative Games and Bankruptcy Problems}

A (TU) game on a finite set of players $N$ is a mapping $v$ associating a real number $v(S)$ with every subset $S \subseteq N$, such that $v(\emptyset)=0$. The subsets $S \subseteq N$ are called coalitions. The real number $v(S)$ represents the worth of coalition $S$, interpreted as the total amount that this coalition can obtain alone, without the rest of the players.

A solution concept is a mapping $\sigma$ associating with each game in a specific class a payoff vector, i.e. a vector $x=\sigma(N, v) \in R^{N}$, whose components represent the payoffs to the players and add up to $v(N)$. Two of the most prominent solution concepts are the Shapley value and the nucleolus.

There is a natural way to associate a game with a bankruptcy problem (see O'Neill, 1982). The worth of a coalition $S$ is what is left, after the other players receive their claims. Thus given a bankruptcy problem $(N, E, c)$ define for every $S \subseteq N$

$$
v_{(N, E, c)}(S)=\left(E-\sum_{k \in N \backslash S} c_{k}\right)_{+} .
$$

Having associated a game to every bankruptcy problem, every solution $\sigma$ for TU games automatically induces a rule for bankruptcy problems, which will also denote by $\sigma$ by abusing notations slightly. It is well known that the Shapley value gives place to the Random Arrival rule, and the nucleolus to the Talmud rule (0'Neill, 1982; and Aumann \& Maschler, 1985, respectively).

The consistency property has been extensively analyzed in the context of cooperative games as well. Several interpretations exist there as well and, just like in bankruptcy problems, also depend on the definition of a reduced situation. It is instructive to compare the consistency property for bankruptcy problems with the one for cooperative games.

Following Davis \& Maschler (1965), given a game $v$ on $N$, a coalition $S$, and a payoff vector $x$, the reduced game $v^{S, x}$ on $S$ is defined as follows

$$
\text { (2) } v^{S, x}(T)= \begin{cases}x(T) & \text { if } T=S \text { or } T=\emptyset, \\ \max \{v(Q \cup T)-x(Q): Q \subseteq N \backslash S\} & \text { if } T \subset S, T \neq \emptyset, S .\end{cases}
$$

A different notion was suggested by Hart \& Mas-Colell (1989). Given a game $v$ on $N$, a coalition $S$, and a TU solution $\sigma$, they define the reduced game $v^{S, \sigma}$ for all $T \subset S$ as

$$
v^{S, \sigma}(T)=v\left(T \cup S^{c}\right)-\sum_{i \in S^{c}} \sigma_{i}\left(T \cup S^{c}, v\right) .
$$


A question arises: do these definitions of a reduced bankruptcy game corresponds to the definitions of reduced bankruptcy problem seen in the previous sections. The answer concerning the definition of Davis \& Maschler for a reduced game (Expression (2)) is given in the following lemma, where it is shown to correspond to the "traditional" reduced bankruptcy problem.

Lemma 2 (Aumann \& Maschler, 1985). Let $(N, E, c)$ be a bankruptcy problem and $\sigma$ a rule such that $x=\sigma(N, E, c)$ with $0 \leq x_{i} \leq c_{i}$. Then for any coalition $S$,

$$
v_{\left(S, E^{S, \sigma}, c_{S}\right)}=v_{(N, E, c)}^{S, x}
$$

Alternatively, it turns out that the definition of a reduced bankruptcy problem introduced in the previous section (Expression (1)) corresponds to the Hart \& Mas-Colell reduced game, but only for the case of bilateral reduced problems, as we show in the next lemma, the proof of which is straightforward and will be omitted.

Lemma 3. Let $(N, E, c)$ be a bankruptcy problem, $\sigma$ a rule, and $S \subseteq N$ such that $|S|=2$, then

$$
v_{\left(S, E^{S, \sigma}, c^{S, \sigma}\right)}=v_{(N, E, c)}^{S, \sigma} .
$$

That is, the following diagrams are commutative
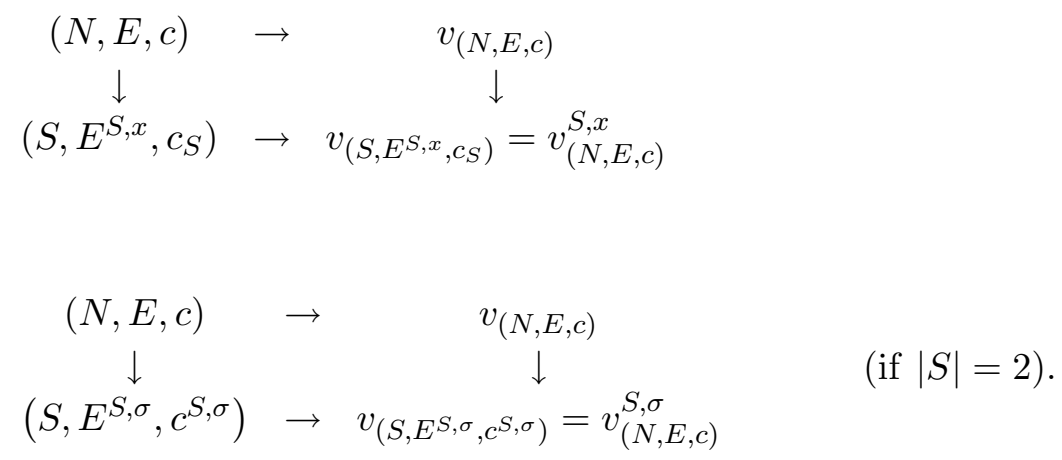

However, if $|S|>2$ the game $v_{(N, E, c)}^{S, \sigma}$ does not correspond in general to a bankruptcy problem. That is, in general the Hart \& Mas-Colell reduced game of a bankruptcy problem is not a game associated to any bankruptcy game, as it is shown in the following example. Let $N=\{1,2,3,4,5\}, E=$ 100, and $c_{i}=40$ for $i=1,2,3,4,5$. Also let $S=\{1,2,3,4\}$, and $\sigma$ a 
symmetric rule. Then

$$
v_{(N, E, c)}^{S, \sigma}(T)= \begin{cases}80 & \text { if } T=S, \\ 35 & \text { if }|T|=3, \\ 40 / 3 & \text { if }|T|=2, \\ 0 & \text { if }|T|=1,\end{cases}
$$

which clearly is not a game associated to any bankruptcy problem. So the second diagram is not necessarily commutative in the many-agent case.

Nevertheless, Theorem 2 suggests a characterization of the Shapley value by means of bilateral consistency à la Hart \& Mas-Colell as follows.

We say that a solution $\sigma$ on TU games satisfies 2- consistency à la Hart $\&$ Mas-Colell (2-CO) if for every game $v$ on $N$, and every $S \subset N$, such that $|S|=2$, it holds

$$
\sigma\left(v^{S, \sigma}\right)=\sigma_{S}(v)
$$

We call a solution $\sigma$ on TU games standard for 2-person games if for every game $v$ on $\{i, j\}$ it holds

$$
\sigma_{i}(v)=v(\{i\})+(1 / 2)(v(\{i, j\})-v(\{i\})-v(\{j\})) .
$$

Theorem 3. The Shapley value, for the class of TU games, is the unique single-point solution concept which is standard for 2-person games and satisfies 2-CO.

Proposition 1 can be easily adapted to prove the uniqueness part of this theorem. The existence part is an immediate corollary of Theorem B in Hart \& Mas-Colell (1989) or Theorem 2 in Maschler \& Owen (1989). Notice finally that the uniqueness in Theorem 3 does not follow from any of these two results since in both works the respective authors use an induction argument on the whole class of games.

\section{REFERENCES}

AUMANN, R. J. and MASCHLER, M. (1985). Game theoretic analysis of a bankruptcy problem from the Talmud. Journal of Economic Theory, 36, 195-213.

DAVIS, M. and MASCHLER, M. (1965). The kernel of a cooperative game. Naval Research Logistic Quarterly, 12, 223-259.

HART, S. and MAS-COLELL, A. (1989). Potential, value and consistency. Econometrica, 57, 589-614. 
LEROUX, J. (2007). A note on consistency in cost-allocation problems. mimeo HEC Montréal.

MASCHLER, M. and OWEN, G. (1989). The consistent Shapley value for hyperplane games. International Journal of Game Theory, 18, 389-407.

O'NEILL, B. (1982). A problem of rights arbitration from the Talmud. Mathematical Social Sciences, 2, 345-371.

SUDHÖLTER, P. (1998). Axiomatizations of Game Theoretical Solutions for One-Output Cost Sharing Problems. Games and Economic Behavior, 24, 142-171.

THOMSON, W. (2003). Axiomatic and Game Theoretic Analysis of Bankruptcy and Taxation Problems: a survey. Mathematical Social Sciences, 45, 249-297.

THOMSON, W. (2006).Consistent allocation rules. Mimeo, University of Rochester. 